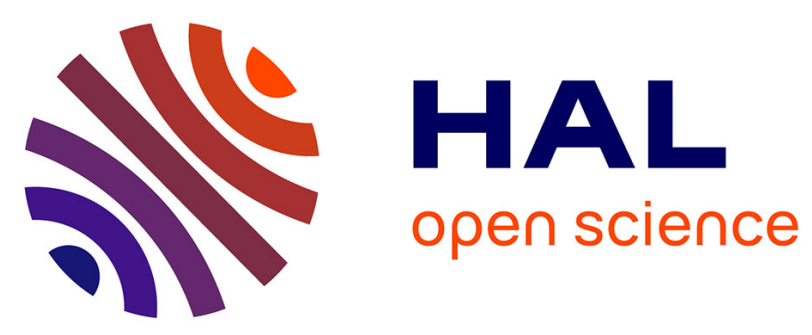

\title{
Looking for Root Hairs to Overcome Poor Soils
}

Thanyakorn Rongsawat, Jean-Benoît Peltier, Jean-Christophe Boyer, Anne-Aliénor Véry, Hervé Sentenac

\section{To cite this version:}

Thanyakorn Rongsawat, Jean-Benoît Peltier, Jean-Christophe Boyer, Anne-Aliénor Véry, Hervé Sentenac. Looking for Root Hairs to Overcome Poor Soils. Trends in Plant Science, 2021, 26 (1), pp.83-94. 10.1016/j.tplants.2020.09.001 . hal-02947957

\section{HAL Id: hal-02947957 https://hal.inrae.fr/hal-02947957}

Submitted on 8 Jan 2021

HAL is a multi-disciplinary open access archive for the deposit and dissemination of scientific research documents, whether they are published or not. The documents may come from teaching and research institutions in France or abroad, or from public or private research centers.
L'archive ouverte pluridisciplinaire HAL, est destinée au dépôt et à la diffusion de documents scientifiques de niveau recherche, publiés ou non, émanant des établissements d'enseignement et de recherche français ou étrangers, des laboratoires publics ou privés.

\section{(ㅇ)(1) $\$$}

Distributed under a Creative Commons Attribution - NonCommercial - NoDerivatives| 4.0 
Dear author,

Please note that changes made in the online proofing system will be added to the article before publication but are not reflected in this PDF.

We also ask that this file not be used for submitting corrections. 


\section{Trends in Plant Science}

1

Q2 Review

Looking for Root Hairs to Overcome Poor Soils

Q4 Q3 Thanyakorn Rongsawat, ${ }^{1,2}$ Jean-Benoît Peltier, ${ }^{1}$ Jean-Christophe Boyer, ${ }^{1}$ Anne-Aliénor Véry,

5

6 and Hervé Sentenac ${ }^{1, \star}$

Breeding new cultivars allowing reduced fertilization and irrigation is a major challenge. International efforts towards this goal focus on noninvasive methodologies, platforms for high-throughput phenotyping of large plant populations, and quantitative description of root traits as predictors of crop performance in environments with limited water and nutrient availability. However, these high-throughput analyses ignore one crucial component of the root system: root hairs (RHs). Here, we review current knowledge on $\mathrm{RH}$ functions, mainly in the context of plant hydromineral nutrition, and take stock of quantitative genetics data pointing at correlations between $\mathrm{RH}$ traits and plant biomass production and yield components.

\section{Root Hairs Enlarge the Soil-Root Interface}

Research efforts aiming at improving understanding of the functioning of root systems are required to better exploit the genetic variation in productivity of crops in poorly fertile soils and to develop new crop cultivars with enhanced capacity for soil resource acquisition $[1,2]$. Root system architecture and, at the root-soil interface, production and elongation of root hairs $(\mathrm{RHs})$, are major determinants of the location and volume of exploited soil, which is why $\mathrm{RHs}$ take center stage in this review. It has been reported that a single rye plant (Secale cereale) can develop more than $10^{10} \mathrm{RHs}$, representing an underground interface of $\sim 400 \mathrm{~m}^{2}$, much larger than that of the aerial parts of the plant [3]. Figure 1 (Key Figure) shows dense and long RHs over almost the whole root system in a 2-week old wheat (Triticum turgidum ssp. durum) seedling. The diameter of the $\mathrm{RH}$ cylinder around the root in the displayed enlargement is approximately ten times larger than that of the root itself and, thus, the volume of this cylinder would be ca. 100 times larger than that of the root. Such a figure indicates that the ability of the root system to take up poorly mobile nutrient ions (e.g., phosphate; see later) can be significantly increased by $\mathrm{RH}$ production. Here, we review some major functions of $\mathrm{RH}$, in the context of plant mineral nutrition, and scrutinize recent attempts to use $\mathrm{RH}$ traits in plant breeding programs.

\section{Adhesion to Soil Particles, Soil Penetration, and Rhizosheath Formation}

$\mathrm{RH}$ s enhance seedling survival upon soil disruption by favoring root anchoring [4]. They also provide grip for root tip penetration in soil. For instance, during germination of maize on a soil displaying a moderate penetrometer resistance, wild-type seedlings took $\sim 16 \mathrm{~h}$ to anchor themselves to the soil, compared with $>30 \mathrm{~h}$ for hairless mutant seedlings, most of which did not become anchored securely [5]. The strength of the grip can be increased by root exudation of adhering molecules [6], as also shown in clinging-climber species, such as English ivy, and their specialized RHs [7]. However, when the resistance to vertical uprooting forces is compared between arabidopsis (Arabidopsis thaliana) wild-type plants and mutant plants impaired in $\mathrm{RH}$ development or lateral root production, the conclusion was that RHs do not contribute to whole-plant anchoring in this operational definition [8].

At a later stage of root system development, $\mathrm{RHs}$ and root exudation of adhesive molecules are involved in rhizosheath (see Glossary) formation [6], which contributes to plant adaptation to abiotic and biotic conditions, as prevention of water loss, nutrient and water acquisition, and

\section{Highlights}

Plant breeding for improved belowground traits, allowing reduced fertilization and irrigation inputs, can contribute to the development of sustainable agriculture practices.

Root hairs ( $\mathrm{RHS}$ ) increase the volume of exploited soil, and have major roles in nutrient and water uptake as well as in beneficial interactions with soil microorganisms.

Plant engagement in mycorrhizal symbiosis also increases the volume of exploited soil, but appears less efficient than $\mathrm{RH}$ development in terms of biomass production in some soil conditions.

Evidence that plant biomass production can be positively correlated to $\mathrm{RH}$ length is available.

${ }^{1}$ Biochimie et Physiologie Moléculaire des Plantes, UMR BPMP, Univ Montpellier, CNRS, INRAE, Montpellier SupAgro, Montpellier 34060, France

${ }^{2}$ Current address: Agricultural Innovation and Management Division, Faculty of Natural Resources, Prince of Songkla University, Hat Yai, Songkhla 90110 Thailand

${ }^{*}$ Correspondence:

herve.sentenac@inrae.fr (H. Sentenac). 


\section{Key Figure}

Q1 Root System of Wheat Seedlings

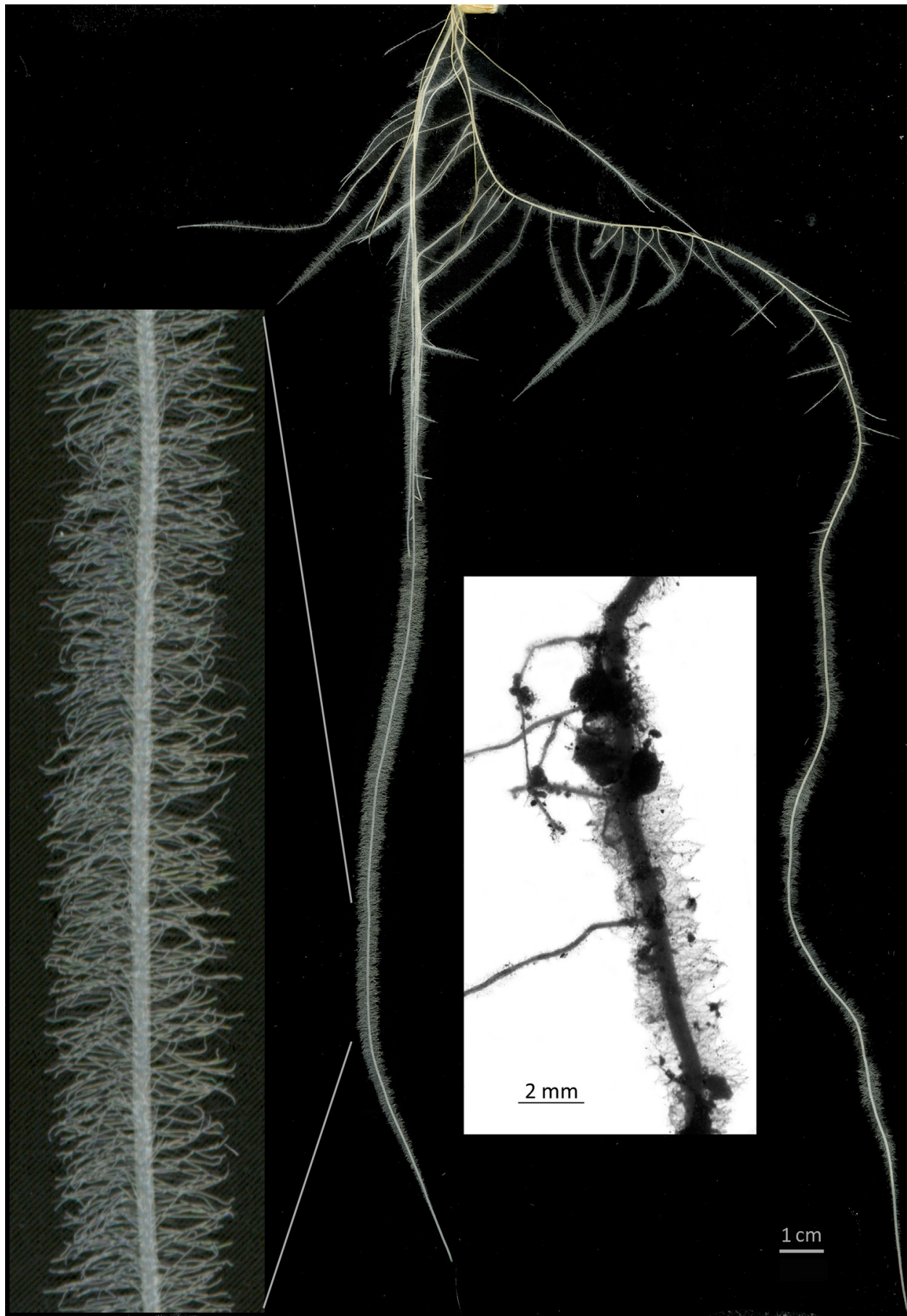

Trends in Plant Science

\section{Glossary}

Rhizosheath: root-soil sheath resulting from agglutination and adhesion to the root of soil particles. Operational

descriptions are proposed from the weight of soil that adheres to roots that have been freshly excavated and submitted to a soil removal treatment (e.g., hand shaking or sonication in water) of standardized vigor $[9,11,12]$. Shaker channels: highly $\mathrm{K}^{+}$-selective voltage-gated channels. The plant Shaker family comprises both hyperpolarization-activated and depolarization-activated channels, involved in $\mathrm{K}^{+}$uptake or $\mathrm{K}^{+}$secretion, respectively. The name 'Shaker' comes from the phenotype of a fly (Drosophila melanogaster) mutant characterized by rapid abnormal movements, in which the first channel of this family was cloned a few years before the cloning of channels of the same type in plants.

Transceptor: acronym of 'transporter' and 'receptor'. A transceptor is endowed with the capacity to mediate membrane transport and the ability to sense and signal the availability of a given solute. 
mechanical defense against herbivorous and plant parasites [9]. A strong correlation has been found between $\mathrm{RH}$ length and rhizosheath weight in wheat [10]. The correlation found in barley (Hordeum vulgare) is weaker [11], and no significant correlation has been observed in 58 other species except for those with quite short RHs [12]. It has been proposed that, when RH length exceeds $\sim 300 \mu \mathrm{m}$, other factors have increasing importance in rhizosheath size and stability, which might include RH density and RH morphology (e.g., bent or hooked forms that would trap more soil, and root and microbial mucilage) $[9,12]$.

\section{Nutrient and Water Acquisition}

Major lines of evidence that RHs contribute significantly to nutrient ion acquisition from the soil can be sorted as follows: (i) nutrient starvation results in increased RH density and length [13,14]; (ii) mutant plants displaying impaired $\mathrm{RH}$ growth show poor nutrient ion uptake and biomass production; furthermore, nutrient accumulation is positively correlated with $\mathrm{RH}$ length under nutrient-deficient conditions [15-18]; (iii) genotypes with longer RHs have been shown in barley and wheat to be better adapted to low nutrient soil $[19,20]$; and (iv) evidence that $\mathrm{RH}$ contribute directly to nutrient uptake has been obtained by various electrophysiological approaches [21-23] or by using dedicated growth devices ensuring that only RHs had access to the nutrient source [24].

Evidence has also been obtained that $\mathrm{RH}$ s can facilitate water uptake $[18,25,26]$. For instance, the absence of RHs affects water absorption and drought tolerance in arabidopsis [18]. In barley, analyses of the relationship between transpiration rate and xylem suction in wild type and hairless mutant plants provided direct evidence that $\mathrm{RH}$ s contribute to water uptake in drying soils in rapidly transpiring plants by increasing the soil-root interface [26]. RHs are also involved in the formation of rhizosheaths, which are more developed in mesophytic grasses in drier conditions [9], which also supports the hypothesis that the control of $\mathrm{RH}$ development has a role in plant adaptation to drought conditions.

\section{Ion Transport Systems at the RH Plasma Membrane}

Molecular analyses, including reverse genetics approaches, most often carried out in arabidopsis, have provided information on the transporters and channels involved in nutrient ion acquisition by roots. Here, we summarize our current understanding regarding potassium $(\mathrm{K})$, nitrogen $(\mathrm{N})$, phosphorus $(\mathrm{P})$, and sulfur $(\mathrm{S})$ acquisition (Figure 2).

$\mathrm{K}^{+}$uptake from the soil by arabidopsis roots is essentially mediated by the high-affinity $\mathrm{K}^{+}$transporter AtHAK5 and the Shaker channels AtAKT1 and AtKC1 [27]. Evidence is available that these three $\mathrm{K}^{+}$transport systems are expressed in $\mathrm{RHs}[15,28]$.

$\mathrm{NO}_{3}{ }^{-}$acquisition by roots involves transporters belonging to three different families: nitrate transporter 1/peptide transporter family (NPF), NRT2, and NRT3 (also named NAR2 for 'nitrate assimilation related family') [29]. The NPF family comprises the extensively studied AtNPF6.3 'transceptor' (endowed with a dual $\mathrm{NO}_{3}{ }^{-}$transport/signaling function [30]), initially named $\mathrm{CHL} 1$ or AtNRT1.1. This membrane protein behaves both as a dual-affinity $\mathrm{NO}_{3}{ }^{-}$transporter and as a $\mathrm{NO}_{3}{ }^{-}$sensor mediating $\mathrm{NO}_{3}{ }^{-}$regulated auxin transport, thereby having an important role in root development [29,31]. Transcriptome data provide evidence that AtNPF6.3/AtNRT1.1 transcripts are present in arabidopsis RHs [32]. The NRT2 family comprises AtNRT2.1 and AtNRT2.2, which physically interact with a member of the NRT3 family, AtNRT3.1 (also named NAR2.1) to form

Figure 1. Main photo: 2-week-old seedling grown in a rhizobox. Inset: part of the root system of a plant grown for 2 months in soil in a pot, showing root hairs in old parts of the root system. Wheat cultivar: Oued Zenati. 


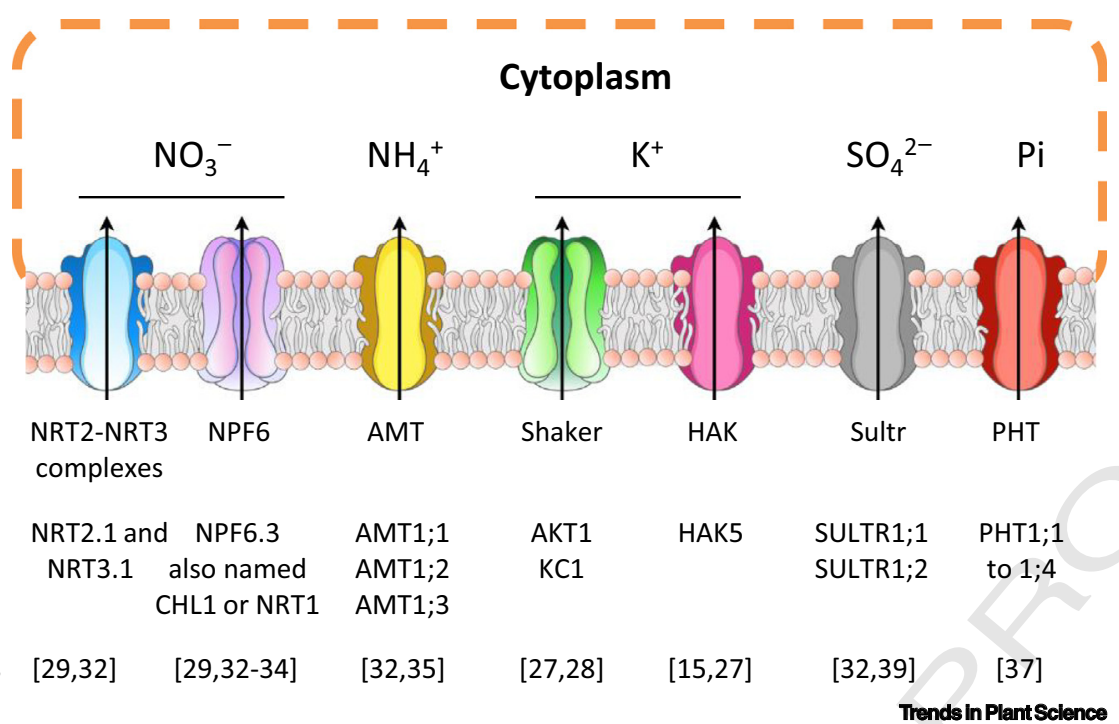

\begin{tabular}{|c|c|c|c|c|c|c|c|}
\hline $\begin{array}{l}\text { Family } \\
\text { name }\end{array}$ & $\begin{array}{l}\text { NRT2-NRT3 } \\
\text { complexes }\end{array}$ & NPF6 & AMT & Shaker & HAK & Sultr & PHT \\
\hline $\begin{array}{l}\text { Major } \\
\text { systems }\end{array}$ & $\begin{array}{l}\text { NRT2.1 and } \\
\text { NRT3.1 }\end{array}$ & $\begin{array}{l}\text { d NPF6.3 } \\
\text { also named } \\
\text { CHL1 or NRT1 }\end{array}$ & $\begin{array}{l}\text { AMT1;1 } \\
\text { AMT1;2 } \\
\text { AMT1;3 }\end{array}$ & $\begin{array}{l}\text { AKT1 } \\
\text { KC1 }\end{array}$ & HAK5 & $\begin{array}{l}\text { SULTR1;1 } \\
\text { SULTR1;2 }\end{array}$ & $\begin{array}{l}\text { PHT1;1 } \\
\text { to } 1 ; 4\end{array}$ \\
\hline References & {$[29,32]$} & {$[29,32-34]$} & {$[32,35]$} & {$[27,28]$} & {$[15,27]$} & {$[32,39]$} & [37] \\
\hline
\end{tabular}

Figure 2. Ion Channels and Transporters Involved in Nutrient Ion Uptake and to Display Expression in Root Hairs in Arabidopsis thaliana. Root hair transcriptome data obtained in Medicago truncatula, Glycine max, Solanum lycopersicum, Zea mays, and Cucumis sativus indicate that close homologs of these arabidopsis channels and transporters are also expressed in root hairs of these dicots and monocots, as well as in those of the lycophyte Selaginella moellendorffii $[32,40]$. The expression of genes encoding high-affinity uptake systems can be repressed in rich media, which might be why expression in root hairs of genes such as AtHAK5 and AtNRT2; 1 is not always revealed by transcriptome analyses of plants grown on rich media while evidenced by other studies using more diluted media (e.g., [15,34]). See [15,27-29,32-35,37,39].

heteromeric structures that contribute significantly to high-affinity $\mathrm{NO}_{3}{ }^{-}$uptake from the soil solution $[29,33]$. AtNRT2.1 and AtNRT3.1 display expression in RHs as shown by reporter gene experiments and/or transcriptome analyses [32,34]. RHs also express members of the AMT, PHT1, and Sultr families, involved in ammonium [35], phosphate [36-38], and sulfate [39] uptake in arabidopsis, respectively [32].

Close homologs of all the above-cited channels and transporters, (AtAKT1, AtKC1, AtHAK5, NPF6.3, AtNRT2.1, and AtNRT3.1) as well as ammonium, phosphate, and sulfate transporters from the AMT, PHT1, and Sultr families, have been identified in Medicago truncatula RH transcriptome data by analyses focused on membrane transport systems [40], and can be found in other RH transcriptomes from both dicots and monocots [32]. Shaker channels and members of the HAK, NRT2, NRT3, AMT, PHT, and Sultr transporter families are also present in the moss Physcomitrella patens [41-44]. Altogether, these observations suggest that major components of the $\mathrm{RH}$ equipment involved in plant mineral nutrition were acquired very early during plant evolution.

The sensitivity of $\mathrm{RH}$ length and density to nutrient and water availability in the soil (see earlier) may involve a role of RHs as sensors of soil conditions. Such a hypothesis of early sensing of water deficit conditions has received support from studies in barley wild type and hairless mutant plants. Analysis of transcriptomes from roots that were sampled at the onset of a water stress revealed that more genes were induced in the roots of the wild-type plants, including, for example, genes involved in abscisic acid biosynthesis [45]. In terms of nutrient sensing, the arabidopsis $\mathrm{NO}_{3}{ }^{-}$ transporter AtNPF6.3/AtNRT1.1 and the Shaker $\mathrm{K}^{+}$channel AtAKT1, which have both been proposed to behave as transceptors, able to sense and signal the availability of their substrates, $\mathrm{NO}_{3}{ }^{-}$and $\mathrm{K}^{+}$, respectively $[30,46]$, are both expressed in $\mathrm{RHs}$ (see earlier). 


\section{RH Production versus Engagement in Mycorrhizal Symbiosis}

Both $\mathrm{RH}$ production and engagement in mycorrhizal symbiosis result in increased soil exploration and exploitation. Arbuscular endomycorrhizal colonization (AM) is associated with either a decrease $[47,48]$ or an increase [49] in RH density and length, depending in some species on the root type (lateral root order) [49]. The decrease has been proposed to result from changes in the root metabolic status and competition for available photosynthates between $\mathrm{RH}$ production and the fungus [47]. The increase has been associated with changes in the expression of auxin metabolism and transport genes, which are likely to impact $\mathrm{RH}$ development [50] (see later). In ectomycorrhizal symbiosis, evidence is available that fungal secretion of the auxin antagonist hypaphorine can inhibit $\mathrm{RH}$ development [51,52].

Mycorrhizal fungal hyphae can have a smaller diameter (ca. $4 \mu \mathrm{m}$ for Glomus intraradices and $5 \mu \mathrm{m}$ for Glomus mosseae [53]) compared with RHs (ca. $10 \mu \mathrm{m}$ in arabidopsis [54]), which allows exploration of smaller soil pores. Furthermore, they extend far beyond the limits of the $\mathrm{RH}$ cylinder. Thus, mycorrhizal symbiosis may be hypothesized to be more efficient for exploiting the soil compared with the promotion of $\mathrm{RH}$ elongation and density. This question has been investigated in barley by comparing wild type and hairless mutant (brb) plants inoculated or not by different endomycorrhizal fungi. Mycorrhizas were found to substitute for RHs in P uptake, but the additional P was most often used less efficiently, in terms of plant growth, compared with $\mathrm{P}$ provided by RHs [55]. A similar study used several barley lines that were differentially affected in $\mathrm{RH}$ development (hairless, short or intermediate, or wild-type RH length phenotypes). The plants were naturally colonized by a live community present in the soil. Endomycorrhizal symbiosis did not fully compensate for the absence of RHs with regard to both P acquisition and biomass production [56]. A third series of similar experiments, using the hairless barley mutant brb and the corresponding wild-type genotype grown under well-watered or drought conditions showed that, with respect to biomass production, endomycorrhizal symbiosis compensated for the absence of RHs in the latter condition but not in the former [57]. Altogether, these reports indicate that AM associations can be less efficient in P-acquisition and biomass production compared with RHs in some environmental conditions.

\section{Organic Compound Exudation, Plant Growth-Promoting Rhizobacteria, and} Stimulation of RH Development

Plants exude large amounts of organic compounds into the soil, rendering the rhizosphere a rich niche for the development of microbial communities. The actual amount of carbon (C) invested in root exudation, which can vary from $5 \%$ to $50 \%$ of the net photosynthesized C $[58,59]$, depends on the health of the plant, its rate of growth, its nutrient and water status, and its microbiota. Root exudation has been compared in wild type and hairless mutant barley plants, revealing that the amount of exuded $\mathrm{C}$ was three times higher in wild-type plants compared with the hairless mutant [60]. Furthermore, experiments carried out with wild type and hairless mutant barley plants showed that an absence of RHs significantly reduced the diversity of the bacterial community [61]. Bacterial attraction by root exudates probably involves selective chemotaxis processes [62].

The microbial community thriving in the rhizosphere can include up to $10^{9}$ bacteria per gram of soil, belonging to diverse taxa $[61,62]$. Within this population, bacteria generically named plant growth promoting rhizobacteria (PGPR) can be recruited by roots to engage in beneficial interactions. PGPR promote plant growth via very diverse mechanisms, such as improved plant mineral nutrition resulting from solubilization of poorly soluble nutrient sources, production of phytohormones that affect root development, and protection against phytoparasites [63]. 
Promotion of $\mathrm{RH}$ development, likely to result in improved mineral nutrition, has been reported in response to diverse PGPR and in various plant species [64-71]. The increase in $\mathrm{RH}$ length can be important, by more than $100 \%[64,68,71]$, making this response to the bacterial inoculation the easiest to detect and, thus, the most straightforward way in laboratory experiments to check whether a given plant species can interact with a given PGPR.

Large differences in the capacity to promote $\mathrm{RH}$ elongation have been observed between PGPR strains [64]. This raises the question of whether such differences are correlated with PGPR capacity to promote plant growth and, thus, are indicative of symbiosis effectiveness. However, pathogenic strains of the bacterium Pseudomonas syringae have been shown to promote $\mathrm{RH}$ elongation in arabidopsis, similar to that seen with beneficial Pseudomonas spp. bacteria [72].

\section{Plasticity of RH Development and Adaptation to External Conditions}

$\mathrm{RH}$ development is strongly responsive to environmental factors, nutrient availability, and rhizosphere microbial communities (see earlier), as well as soil porosity, strength, and water content [73]. Different patterns of $\mathrm{RH}$ distribution within the root epidermis can be identified among plant species (Box 1) but the question of whether a given pattern has specific advantages and in what environmental conditions is poorly documented.

Studies in arabidopsis ( $\mathrm{RH}$ distribution pattern 3; Box 1) to investigate how external biotic or abiotic conditions can impact epidermal cell differentiation and $\mathrm{RH}$ morphogenesis, have brought to light hormone-driven processes [74]. Evidence has been obtained that auxin and ethylene have major roles in the control of epidermal cell differentiation and $\mathrm{RH}$ development and that the pathways allowing these two hormones to affect $\mathrm{RH}$ formation are significantly congruent [54,74-77]. Proper auxin distribution is required for correct cell fate assignment and $\mathrm{RH}$ formation (both initiation site selection and tip growth). In arabidopsis, auxin regulates $\mathrm{RH}$ formation by acting downstream of RHD6, and probably primarily via RSL4 [78,79], two transcription factors with central roles in $\mathrm{RH}$ development (Box 2). Ethylene also acts on $\mathrm{RH}$ formation downstream of RHD6 [78]. Evidence is also available that jasmonic acid, strigolactones, and cytokinins are positive regulators of $\mathrm{RH}$ growth, whereas brassinosteroids and abscisic acid are negative regulators $[54,74,75,80]$.

The widely reported increase in $\mathrm{RH}$ density and length in response to low $\mathrm{P}$ availability involves induction of $\mathrm{RH}$-expressed auxin-inducible transcription factors, including RSL2 and RSL4 [81] and ethylene-mediated events with a role in the control of $\mathrm{RH}$ gene expression [82]. Promotion of RH development by PGPR species has also been shown to involve auxin [71], ethylene [65], and a complex interplay of auxin and ethylene signaling pathways [83]. Nevertheless, a case of PGPR-induced promotion of $\mathrm{RH}$ elongation poorly dependent on auxin and ethylene signaling mechanisms has been reported in arabidopsis [67].

Finally, although control of $\mathrm{RH}$ longevity is likely to be of major importance in root system adaptation to soil abiotic and biotic factors, $\mathrm{RH}$ longevity is still poorly documented. Reported values in barley vary from a few days to 2-3 weeks [84-86]. Evidence has been obtained that apoptosis-like programmed cell death (AL-PCD), characterized by protoplast retraction, nuclear DNA fragmentation, and sensitivity to inhibitors of caspase-3-like activity, occurs in arabidopsis $\mathrm{RH}$ in response to heat shock, salt stress, and reactive oxygen species $\left(\mathrm{ROS} ; \mathrm{H}_{2} \mathrm{O}_{2}\right)$ treatment $[87,88]$. Basal AL-PCD rates ranging from ca. $5 \%$ to $15 \%$ have been monitored in arabidopsis seedlings classically grown on agar plates [88]. Thus, it is tempting to hypothesize that the extension of functional $\mathrm{RH}$ zones might be regulated by AL-PCD in response to local environmental conditions. 


\section{Box 1. Root Hair Distribution Patterns}

$\mathrm{RH}$ cell distribution within the root epidermis varies among angiosperms. The distribution patterns have been sorted into three basic types: random, alternating, and position-dependent, named types 1, 2, and 3, respectively [106,107] (Figure I). In type 1 development, displayed by, for example, Medicago truncatula, barley, and maize, $\mathrm{RH}$ cells can differentiate from any epidermal cell. This results in the absence of regular patterns, in contrast to types 2 and 3 development. Type 2 , displayed by, for example, wheat, rice, and Brachypodium, involves asymmetry in the last cell division just before epidermal cells leave the meristematic zone, leading to the formation of two daughter cells that differ in size. Only the smaller cell differentiates into an $\mathrm{RH}$ cell. Thus, in each epidermal cell file along the root longitudinal axis, $\mathrm{RH}$ cells and non-hair cells alternate. In type 3 plants, such as Arabidopsis thaliana and Brassica, cell files comprising entirely RHs along the root longitudinal axis alternate with one or more non-hair cell files. Evidence has been obtained in Arabidopsis that this pattern results from position-dependent hair cell specification: $\mathrm{RH}$ cells are located over two underlying cortical cells (the $\mathrm{H}$ cell position), whereas non-hair cells are positioned over a single cortical cell (the $\mathrm{N}$ cell position; see Box 2 in the main text) [54]. However, this classification of RH distribution patterns into three major types do not describe the whole diversity regarding this trait since, for instance, a type 3 variant, in which long hairs differentiate from cells in $\mathrm{H}$ position and short hairs from cells in the $\mathrm{N}$ position, has been described [108].

\section{Type 1}

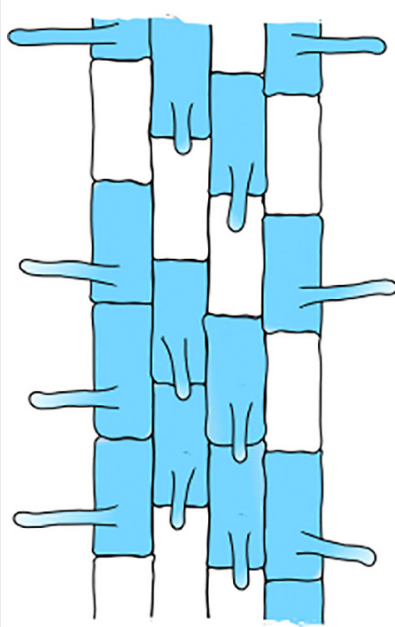

Type 2

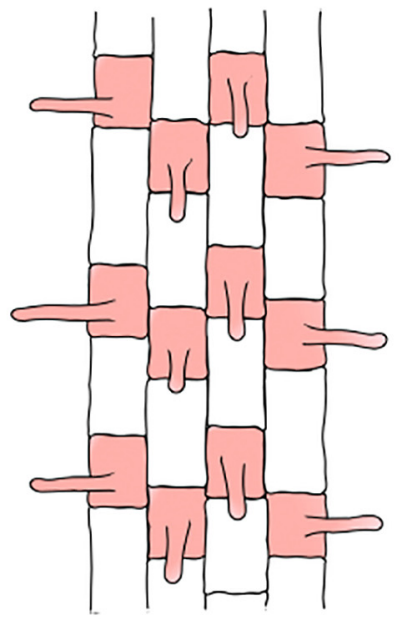

Type 3

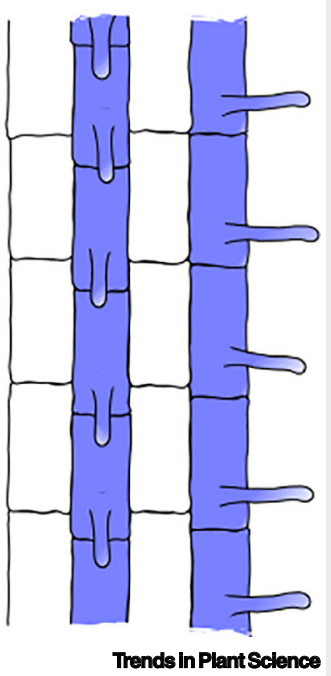

Trends in Plant Science

Figure I. Root Hair Distribution Patterns.

\section{Quantitative Trait Loci of RH Production and Plant Yield}

Quantitative trait loci (QTLs) of RH length have been identified in various crops (e.g., barley [89], maize [90], and wheat [10,91-93]). A seminal study of the correlation between $\mathrm{RH}$ length and biomass production and yield, reported by Gahoonia and Nielsen [19], explored the biological diversity within 38 barley cultivars. Large variations in $\mathrm{RH}$ length, from $\sim 0.4 \mathrm{~mm}$ to $>1.3 \mathrm{~mm}$, were observed in hydroponically grown plants, consistent with variations thereafter observed in field conditions. Then, a set of ten representative cultivars was tested in field experiments, with different levels of soil $P$ availability. The complete set of results indicated that barley genotypes with long RHs displayed higher tolerance to low $\mathrm{P}$ conditions, and expressed higher yield potentials both in low and high P soils [19]. In a similar experiment, characterization of barley mutant lines with various $\mathrm{RH}$ phenotypes, screened from a mutagenized population, showed that $\mathrm{RH}$ length was important for shoot $\mathrm{P}$ accumulation and biomass production, especially under combined water and phosphorus stress [94]. However, for grain yield, only the presence of RHs, and not RH length, was critical. The difference in $\mathrm{RH}$ length between the genotypes classified as 'Short RH' and 'Long RH' (0.54 mm vs $0.69 \mathrm{~mm}$ ) in this report was small compared with the differences observed within the set of barley cultivars previously used by Gahoonia and Nielsen 


\section{Box 2. Arabidopsis Root Hair Development}

Figure I describes the core of the current model of the regulation of $\mathrm{RH}$ development by the intrinsic developmental program in arabidopsis [54,75], which displays a type-3 RH distribution pattern (see Box 1 in the main text). The default fate for an epidermal cell is an $\mathrm{RH}$ cell, and entry in the non-hair cell developmental program involves expression of a transcription factor (TF) named GL2 (GLABRA2), which ultimately blocks the hair pathway. In a given epidermal cell, the level of GL2 expression, and thereby cell fate, is determined by the relative position of this cell with respect to the underlying cortical cells. An epidermal cell in contact with two cortical cells, a position which is named 'H' (for 'hair'), develops into an $\mathrm{RH}$ cell, whereas a cell in contact with a single cortical cell, a position named ' $\mathrm{N}$ ' (for 'non hair'), enters the non-hair cell developmental program. Signals leading to this differentiation pattern are emitted by the cortical cell layer, and the signaling pathway involves JACKDAW (JKD), a zinc finger protein expressed in cortical cells, and SCRAMBLED (SCM), an RH plasmalemma receptor-like kinase. Perception of the signal together with a highly diversified and complex series of mechanisms contributes to tune the relative abundancy in $\mathrm{N}$ and $\mathrm{H}$ cells of two TFs, WER and CPC. Each of these TFs is able to form a complex with three other TFs, GL3, EGL3, and TTG1. In N cells, due to a larger abundancy of WER, a WER-GL3/EGL3-TTG1 complex is formed and activates the expression of GL2, which blocks the hair fate by inhibiting the expression of TFs, among which RHD6, required for RH formation. In H cells, CPC inhibits the function of the WERGL3/EGL3-TTG1 complex by interfering with WER binding to GL3/EGL3 in a competitive manner, thereby leading to a CPC inactive complex with respect to GL2 expression. Absence of the block by GL2 allows expression of RHD6 and other TFs (RSL1 and RSL2) with major roles in RH initiation and elongation. The TF RSL4 is one of the direct targets of RHD6 and a major contributor to the expression of $\mathrm{RH}$ cell-specific genes involved in $\mathrm{RH}$ elongation.

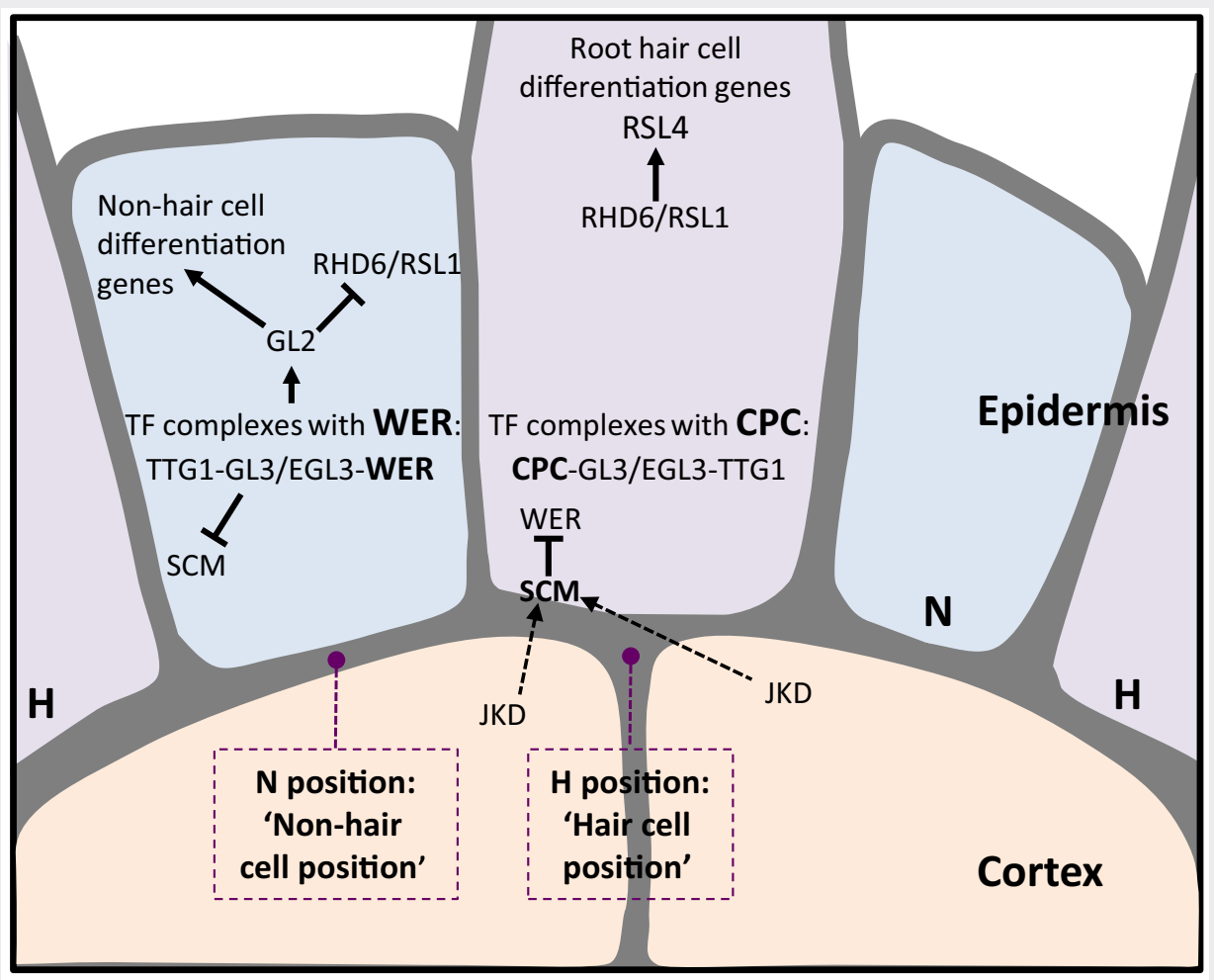

Trends in Plant Science

Figure I. Arabidopsis Root Hair Development. Arrows indicate positive control. Blunted lines indicate negative regulation. Broken lines indicate intercellular signal movement.

[19]. Evidence that plant biomass production can be positively correlated to $\mathrm{RH}$ length in barley has also been provided by phenotyping 175 lines from a doubled-haploid (DH) population, using rhizosheath size as a surrogate for $\mathrm{RH}$ length and the normalized difference vegetation index (NDVI) as a surrogate for crop biomass. These analyses revealed colocation between QTLs for root rhizosheath size and NDVI-estimated biomass [89]. QTLs for RH length have 
also been found to colocate with QTL for yield components in wheat [91]. However, further work is still required to investigate whether, and under what environmental conditions, longer $\mathrm{RHs}$ benefit grain yield.

\section{Concluding Remarks and Future Perspectives}

$\mathrm{RH}$ s have major roles in plant autotrophy and fitness by contributing to the anchorage of growing root tips into the soil, to soil mining for water and nutrient acquisition, and to interactions with soil microorganisms. The RH cell model is extensively used to decipher processes of cell fate and celltype patterning in plants as well as the mechanisms involved in tip growth. However, it is clear that many questions regarding $\mathrm{RH}$ development and functions at the soil-root interface deserve to be further investigated, such as the mechanisms involved in epidermal cell fate in species displaying a RH patterning different from that in arabidopsis (Box 1), or the environmental and internal determinants of $\mathrm{RH}$ longevity and apoptosis-like programmed cell death.

In terms of crop breeding, evidence is available that the genetic variation in root system and $\mathrm{RH}$ traits can be used to reduce fertilization and irrigation inputs and increase crop yield in poor soil conditions $[95,96]$. With the objective of contributing to a vital new green revolution, strong efforts have been aimed at developing methodologies and imaging platforms for high-throughput phenotyping (HTP) of root trait variation in large genotype collections to identify promising germplasm and markers for selection. So far, the root traits that have been analyzed by such HTP approaches and shown to be positively associated with yield in field experiments, at least in some soil and environmental conditions, most often correspond to macroscopic features, such as 'primary root length' in oilseed rape [97], or 'seminal root number', 'total root length' [98], 'narrow root angle' (thought to result in a steep root phenotype) [99], or 'root diameter' [100] in wheat. None of the HTP methodologies used in these studies were reported to have included $\mathrm{RH}$ development in the targeted root traits, probably because the phenotyping procedure was not aimed at obtaining and analyzing high-resolution images. $\mathrm{RH}$ traits that have to be taken into account are length and density as well as the sensitivity of these parameters to abiotic and biotic conditions, such as reduced nutrient and water availability or presence of PGPR. It is also likely that the location and relative extension of the root zones bearing live $\mathrm{RH}$ s are important traits. HTP methodologies could also be used to screen, for instance, collections of crop cultivars together with collections of beneficial soil bacteria by phenotyping the $\mathrm{RH}$ responses to the inoculated bacteria before assessing the effects of selected bacterial partners on plant growth in soil conditions. Various low-cost HTP methodologies can be used/adapted for such research objectives, including the so-called 'paper-roll' and 'pouch and wick' setups and other 2D phenotyping methodologies [101]. Our group is currently developing a similar 2D HTP approach using rhizobox-like devices in which root growth occurs (Figure 1) on a piece of non-putrescible tissue under automatically controlled watering. More complex 3D phenotyping methodologies could also be developed using heterogeneous transparent substrates that mimic soil features $[102,103]$.

$\mathrm{RH}$ phenotyping in plants grown in field conditions can provide valuable criteria for plant breeding programs. Gentle and careful washing of excavated root systems can allow quantification of RH traits, namely length and density [3,104], but this is poorly amenable to high-throughput procedures. By contrast, such analyses of $\mathrm{RH}$ genotypic variation in the field have provided information in line with the results of HTP analyses (paper-roll type) and have been found to be effective in breeding programs for edaphic stress tolerance in low-input agriculture $[95,104]$. Thus, evidence is already available that a combination of HTP approaches with assessment of the selected germplasm in field conditions can increase the efficiency and speed up plant breeding for lowinput agriculture $[95,96,105]$. This suggests that further development/adaptation of HTP
What are the sensing and signaling mechanisms allowing $\mathrm{RH}$ production and elongation to respond to soil features (nutrient ion availability, soil moisture, texture, porosity...)?

How long do RHs stay alive and functional in the soil and what are the internal and external determinants of $\mathrm{RH}$ lifespan?

Do the different $\mathrm{RH}$ distribution patterns have specific physiological advantages and under what environmental conditions?

What are the comparative costbenefit ratios of $\mathrm{RH}$ production and mycorrhization, in terms of biomass production and grain yield?

What is the physiological meaning of the stimulation of $\mathrm{RH}$ elongation by rhizobacteria, and can this stimulation be operationally considered, in HTP methodologies, as an indication of an engagement in beneficial interactions?

What kinds of high-throughput root trait phenotyping methodologies could take into RHs consideration?

What have been the consequences of generations of selective breeding for increased yields in artificial soil conditions on the ability of plants to invest photosynthates in root development and functions? 
303

310

311

312

313

316

317

318

319

320

321

322

323

324

325

326

328

329

332

333

334

335

336

337

338

339

340

341

342

343

344

345

346

347

348

349

350

351

352

353

354

355

356

357

358

359

360

361

362

363

364

365

366

367

368

369

370

372

methodologies enabling quantitative phenotyping of $\mathrm{RH}$ traits and of their responses to abiotic and biotic conditions would contribute significantly to such programs.

\section{Acknowledgments}

We thank Martin Boeglin for help in preparing Figure 1. This work was supported by the French National Research Agency (ANR) [ANR-11-BSV7-010-02 (to H.S. and A-A.V.) and ANR ANR-19-CE32-0011 (to J-B.P.)], an EU Erasmus Mundus grant, Alfabet project (to T.R.) and an ERANET EU Arimnet2 grant (no. 618127) (to H.S.).

\section{Resources}

i https://phytozome.jgi.doe.gov/pz/portal.htm|\#l!info?alias=Org_Ppatens

\section{References}

1. Lynch, J.P. (2019) Root phenotypes for improved nutrient capture: an underexploited opportunity for global agriculture. New Phytol. 223, 548-564

2. Zhu, Y.H. et al. (2019) Evolutionary agroecology: trends in root architecture during wheat breeding. Evol. Appl. 12, 733-743

3. Dittmer, H.J. (1937) A quantitative study of the roots and root hairs of a winter rye plant (Secale cereale). Am. J. Bot. 24, 417-420

4. Choi, H.-S. et al. (2019) Root hairs enhance Arabidopsis seedling survival upon soil disruption. Sci. Rep. 9, 11181

5. Bengough, A.G. et al. (2016) Root hairs aid soil penetration by anchoring the root surface to pore walls. J. Exp. Bot. 67 , 1071-1078

6. Galloway, A.F. et al. (2020) Cereal root exudates contain highly structurally complex polysaccharides with soil-binding properties. Plant J. 103, 1666-1678

7. Huang, Y. et al. (2016) Nanospherical arabinogalactan proteins are a key component of the high-strength adhesive secreted by English ivy. Proc. Natl. Acad. Sci. U. S. A. 113, E3193-E3202

8. Bailey, P.H. et al. (2002) The role of root system architecture and root hairs in promoting anchorage against uprooting forces in Allium cepa and root mutants of Arabidopsis thaliana. J. Exp. Bot. 53, 333-340

9. Pang, J. et al. (2017) Unwrapping the rhizosheath. Plant Soil 418, 129-139

0. Delhaize, E. et al. (2015) The genetics of rhizosheath size in a multiparent mapping population of wheat. J. Exp. Bot. 66 , 4527-4536

11. George, T.S. et al. (2014) Understanding the genetic contro and physiological traits associated with rhizosheath production by barley (Hordeum vulgare). New Phytol. 203, 195-205

12. Brown, L.K et al. (2017) The rhizosheath - a potential trait for future agricultural sustainability occurs in orders throughout the angiosperms. Plant Soil 418, 115-128

13. Bates, T.R. and Lynch, J.P. (1996) Stimulation of root hair elongation in Arabidopsis thaliana by low phosphorus availability. Plant Cell Environ. 19, 529-538

14. Janes, G. et al. (2018) Cellular patterning of Arabidopsis roots under low phosphate conditions. Front. Plant Sci. 9, 735

5. Ahn, S.J. et al. (2004) Expression of KT/KUP genes in Arabidopsis and the role of root hairs in $\mathrm{K}^{+}$uptake. Plant Physiol. $134,1135-1145$

16. Canales, J. et al. (2017) Nitrate induction of root hair density is mediated by TGA1/TGA4 and CPC transcription factors in Arabidopsis thaliana. Plant J. 92, 305-316

17. Haling, R.E. et al. (2013) Root hairs improve root penetration, root-soil contact, and phosphorus acquisition in soils of different strength. J. Exp. Bot. 64, 3711-3721

18. Tanaka, N. et al. (2014) Characteristics of a root hair-less line of Arabidopsis thaliana under physiological stresses. J. Exp. Bot. $65,1497-1512$

19. Gahoonia, T.S. and Nielsen, N.E. (2004) Barley genotypes with ong root hairs sustain high grain yields in low-P field. Plant Soil 262, 55-62

20. Klinsawang, S. (2018) Effects of root hair length on potassium acquisition in rice (Oryza sativa I.). Appl. Ecol. Environ. Res. 16, $1609-1620$
21. Babourina, O. et al. (2001) $\mathrm{K}^{+}$transport by Arabidopsis root 373 hairs at low pH. Funct. Plant Biol. 28, 637 374

22. Lew, R.R. (1998) Immediate and steady state extracellular 375 ionic fluxes of growing Arabidopsis thaliana root hairs under 376 hyperosmotic and hypoosmotic conditions. Physiol. Plant. 377 104, 397-404

Meharg, A.A. and Blatt, M.R. (1995) $\mathrm{NO}_{3}^{-}$transport across the 379 plasma membrane of Arabidopsis thaliana root hairs: kinetic 380 control by $\mathrm{pH}$ and membrane voltage. J. Membr. Biol. 145, 381 49-66 382

24. Gahoonia, T.S. and Nielsen, N.E. (1998) Direct evidence on 383 participation of root hairs in phosphorus $\left.{ }^{32} \mathrm{P}\right)$ uptake from 384 soil. Plant Soil 198, 147-152 385

25. Ahmed, M.A. et al. (2018) Hydraulic processes in roots and the 386 rhizosphere pertinent to increasing yield of water-limited grain 387 crops: a critical review. J. Exp. Bot. 69, 3255-3265 388

26. Carminati, A. et al. (2017) Root hairs enable high transpiration 389 rates in drying soils. New Phytol. 216, 771-781 390

27. Véry, A.A. et al. (2014) Molecular biology of $\mathrm{K}^{+}$transport across 391 the plant cell membrane: what do we learn from comparison 392 be6 ween plant species. J. Plant Physiol. 171, 748-769 393

28. Reintanz, B. et al. (2002) AtKC1, a silent Arabidopsis potas- 394 sium channel alpha -subunit modulates root hair $\mathrm{K}^{+}$influx. 395 Proc. Natl. Acad. Sci. U. S. A. 99, 4079-4084 396

29. Wang, Y.Y. et al. (2018) Nitrate transport, signaling, and use 397 efficiency. Annu. Rev. Plant Biol. 69, 85-122 398

30. Gojon, A. et al. (2011) Nitrate transceptor(s) in plants. J. Exp. 399 Bot. 62, 2299-2308 400

31. Krouk, G. et al. (2010) Nitrate-regulated auxin transport by 401 NRT1.1 defines a mechanism for nutrient sensing in plants. 402 Dev. Cell 18, 927-937 403

32. Huang, L. et al. (2017) Diversification of root hair development 404 genes in vascular plants. Plant Physiol. 174, 1697-1712 405

33. Yong, Z. et al. (2010) Characterization of an intact two- 406 component high-affinity nitrate transporter from Arabidopsis 407 roots. Plant J. 63, 739-748 408

34. Nazoa, P. et al. (2003) Regulation of the nitrate transporter 409 gene AtNRT2.1 in Arabidopsis thaliana: responses to nitrate, 410 amino acids and developmental stage. Plant Mol. Biol. 52, 411 689-703 412

35. Yuan, L. et al. (2007) The organization of high-affinity ammonium 413 uptake in Arabidopsis roots depends on the spatial arrangement 414 and biochemical properties of AMT1-type transporters. Plant Cell 415 19, 2636-2652 416

36. Młodzińska, E. and Zboińska, M. (2016) Phosphate uptake and 417 allocation - a closer look at Arabidopsis thaliana L. and Oryza 418 sativa L. Front. Plant Sci. 7, $1198 \quad 419$

37. Nussaume, L. et al. (2011) Phosphate import in plants: focus 420 on the PHT1 transporters. Front. Plant Sci. 2, $83 \quad 421$

38. Wang, F. et al. (2018) Molecular mechanisms of phosphate 422 transport and signaling in higher plants. Semin. Cell Dev. Biol. 423 74, 114-122 424

39. Takahashi, H. (2019) Sulfate transport systems in plants: 425 functional diversity and molecular mechanisms underlying reg- 426 ulatory coordination. J. Exp. Bot. 70, 4075-4087 427

40. Damiani, I. et al. (2016) Nod factor effects on root hair-specific 428 transcriptome of Medicago truncatula: focus on plasma 429 
membrane transport systems and reactive oxygen species networks. Front. Plant Sci. 7, 794

41. De Michele, R. et al. (2012) Ammonium and urea transporter inventory of the Selaginella and Physcomitrella genomes. Front. Plant Sci. 3, 62

42. Garciadeblás, B. et al. (2007) Potassium transport systems in the moss Physcomitrella patens: pphak1 plants reveal the complexity of potassium uptake. Plant J. 52, 1080-1093

43. Takahashi, H. et al. (2012) Evolutionary relationships and functional diversity of plant sulfate transporters. Front. Plant Sci. 2 119-128

44. Tsujimoto, R. et al. (2007) Distinct roles of nitrate and nitrite in regulation of expression of the nitrate transport genes in the moss Physcomitrella patens. Plant Cell Physiol. 48, 484-497

45. Kwasniewski, M. et al. (2016) Transcriptome analysis reveals the role of the root hairs as environmental sensors to maintain plant functions under water-deficiency conditions. J. Exp. Bot. 67, 1079-1094

46. Shahzad, Z. and Amtmann, A. (2017) Food for thought: how nutrients regulate root system architecture. Curr. Opin. Plant Biol. 39, 80-87

47. Orfanoudakis, M. et al. (2010) Both the arbuscular mycorrhiza fungus Gigaspora rosea and Frankia increase root system branching and reduce root hair frequency in Alnus glutinosa. Mycorrhiza 20, 117-126

48. Sun, X.-G. and Tang, M. (2013) Effect of arbuscular mycorrhizal fungi inoculation on root traits and root volatile organic compound emissions of Sorghum bicolor. S. Afr. J. Bot. 88 373-379

49. Wu, Q.S. et al. (2016) Mycorrhiza alters the profile of root hairs in trifoliate orange. Mycorrhiza 26, 237-247

50. Liu, C.Y. et al. (2018) Mycorrhiza stimulates root-hair growth and IAA synthesis and transport in trifoliate orange under drought stress. Sci. Rep. 8, 1978

51. Ditengou, F.A. et al. (2000) Root hair elongation is inhibited by hypaphorine, the indole alkaloid from the ectomycorrhizal fungus Pisolithus tinctorius, and restored by indole-3-acetic acid. Planta 211, 722-728

52. Rigas, S. et al. (2013) Root gravitropism and root hair development constitute coupled developmental responses regulated by auxin homeostasis in the Arabidopsis root apex. New Phytol. 197, 1130-1141

53. Drew, E.A. et al. (2003) Beyond the rhizosphere: growth and function of arbuscular mycorrhizal external hyphae in sands of varying pore sizes. Plant Soil 251, 105-114

54. Grierson, C. et al. (2014) Root hairs. Arabidopsis Book 12 , e0172

55. Jakobsen, I. et al. (2005) Contrasting phosphate acquisition of mycorrhizal fungi with that of root hairs using the root hairless barley mutant. Plant Cell Environ. 28, 928-938

56. Brown, L.K. et al. (2013) Interactions between root hair length and arbuscular mycorrhizal colonisation in phosphorus deficient barley (Hordeum vulgare). Plant Soil 372, 195-205

57. Li, T. et al. (2014) Relative importance of an arbuscular mycorrhizal fungus (Rhizophagus intraradices) and root hairs in plant drought tolerance. Mycorrhiza 24, 595-602

58. Haichar, F.E.Z. et al. (2016) Stable isotope probing of carbon flow in the plant holobiont. Curr. Opin. Biotechnol. 41, 9-13

59. Venturi, V. and Keel, C. (2016) Signaling in the rhizosphere. Trends Plant Sci. 21, 187-198

60. Holz, M et al. (2018) Root hairs increase rhizosphere extension and carbon input to soil. Ann. Bot. 121, 61-69

61. Robertson-Albertyn, S. et al. (2017) Root hair mutations displace the barley rhizosphere microbiota. Front. Plant Sci. 8,1094

62. Bulgarelli, D. et al. (2013) Structure and functions of the bacterial microbiota of plants. Annu. Rev. Plant Biol. 64, 807-838

63. Vacheron, J. et al. (2013) Plant growth-promoting rhizobacteria and root system functioning. Front. Plant Sci. 4, 356

64. Contesto, C. et al. (2008) Effects of rhizobacterial ACC deaminase activity on Arabidopsis indicate that ethylene mediates local root responses to plant growth-promoting rhizobacteria. Plant Sci. 175, 178-189

65. Galland, M. et al. (2012) The ethylene pathway contributes to root hair elongation induced by the beneficial bacteria
Phyllobacterium brassicacearum STM196. Plant Sci. 190, 505 74-81 506

66. Jain, D.K. and Patriquin, D.G. (1984) Root hair deformation, 507 bacterial attachment, and plant growth in wheat-Azospirillum 508 associations. Appl. Environ. Microbiol. 48, 1208-1213 509

67. López-Bucio, J. et al. (2007) Bacillus megaterium rhizobacteria 510 promote growth and alter root-system architecture through an 511 auxin- and ethylene-independent signaling mechanism in 512 Arabidopsis thaliana. Mol. Plant-Microbe Interact. 20, 207-217 513

68. Poitout, A et al. (2017) Local signalling pathways regulate the 514 Arabidopsis root developmental response to Mesorhizobium 515 loti inoculation. J. Exp. Bot. 68, 1199-1211 516

69. Ribaudo, C.M et al. (2006) Azospirillum sp. promotes root hair 517 development in tomato plants through a mechanism that 518 involves ethylene. J. Plant Growth Regul. 25, 175-185 519

70. Vacheron, J. et al. (2018) Differential contribution of plant- 520 beneficial functions from Pseudomonas kilonensis F113 521 to root system architecture alterations in Arabidopsis 522 thaliana and Zea mays. Mol. Plant-Microbe Interact. 31, 523 212-223 524

71. Zamioudis, C. et al. (2013) Unraveling root developmental pro- 525 grams initiated by beneficial Pseudomonas spp. bacteria. Plant 526 Physiol. 162, 304-318 527

72. Pecenková, T. et al. (2017) Early Arabidopsis root hair growth 528 stimulation by pathogenic strains of Pseudomonas syringae. 529 Ann. Bot. 120, 437-446 530

73. Haling, R.E. et al. (2014) Root hair length and rhizosheath mass 531 depend on soil porosity, strength and water content in barley 532 genotypes. Planta 239, 643-651 533

74. Vissenberg, K. et al. (2020) Hormonal regulation of root hair 534 growth and responses to the environment in Arabidopsis. 535 J. Exp. Bot. 71, 2412-2427 536

75. Cui, S. et al. (2018) Regulation and functional diversification of 537 root hairs. Semin. Cell Dev. Biol. 83, 115-122 538

76. Bruex, A. et al. (2012) A gene regulatory network for root 539 epidermis cell differentiation in Arabidopsis. PLoS Genet. 8, 540 e1002446 541

77. Zhang, S. et al. (2016) Multiple phytohormones promote root 542 hair elongation by regulating a similar set of genes in the root 543 epidermis in Arabidopsis. J. Exp. Bot. 67, 6363-6372 544

78. Masucci, J.D. and Schiefelbein, J.W. (1996) Hormones act 545 downstream of TTG and GL2 to promote root hair outgrowth 546 during epidermis development in the Arabidopsis root. Plant 547 Cell 8, 1505-1517

79. Yi, K. et al. (2010) A basic helix-loop-helix transcription factor 549 controls cell growth and size in root hairs. Nat. Genet. 42, 550 264-267

80. Han, G. et al. (2020) Arabidopsis ZINC FINGER PROTEIN1 552 acts downstream of GL2 to repress root hair initiation and 553 elongation by directly suppressing bHLH genes. Plant Cell 554 32, 206-225 555

81. Bhosale, R. et al. (2018) A mechanistic framework for auxin 556 dependent Arabidopsis root hair elongation to low external 557 phosphate. Nat. Commun. 9, 1409

82. Song, L. et al. (2016) The molecular mechanism of ethylene- $\mathbf{5 5 9}$ mediated root hair development induced by phosphate 560 starvation. PLoS Genet. 12, e1006194 561

83. Poupin, M.J. et al. (2016) A complex molecular interplay 562 of auxin and ethylene signaling pathways Is involved in 563 Arabidopsis growth promotion by Burkholderia phytofirmans 564 PsJN. Front. Plant Sci. 7, 492

84. Henry, C.M. and Deacon, J.W. (1981) Natural (non-pathogenic) 566 death of the cortex of wheat and barley seminal roots, as evi- 567 denced by nuclear staining with acridine orange. Plant Soil 60, 568 255-274 569

85. Holden, J. (1975) Use of nuclear staining to assess rates of $\mathbf{5 7 0}$ cell death in cortices of cereal roots. Soil Biol. Biochem. 7, 571 333-334 572

86. McElgunn, J.D. and Harrison, C.M. (1969) Formation, elongation, 573 and longevity of barley root hairs. Agron. J. 61, 79-81 574

87. Hogg, B.V. et al. (2011) An in vivo root hair assay for determin- 575 ing rates of apoptotic-like programmed cell death in plants. 576 Plant Methods 7, 45

88. Tan, K. et al. (2016) Nuclear dynamics and programmed cell 578 death in Arabidopsis root hairs. Plant Sci. 253, 77-85 579 
89. Gong, X. and McDonald, G. (2017) QTL mapping of root traits in phosphorus-deficient soils reveals important genomic regions for improving NDVI and grain yield in barley. Theor Appl. Genet. 130, 1885-1902

90. Zhu, J. et al. (2005) Mapping of QTL controlling root hair length in maize (Zea mays L.) under phosphorus deficiency. Plant Soil 270, 299-310

91. Horn, R. et al. (2016) Mapping of quantitative trait loci for root hair length in wheat identifies loci that co-locate with loci for yield components. J. Exp. Bot. 67, 4535-4543

92. James, R.A. et al. (2016) Rhizosheaths on wheat grown in acid soils: phosphorus acquisition efficiency and genetic control. J. Exp. Bot. 67, 3709-3718

93. Liu, M. et al. (2017) Analysis of aneuploid lines of bread wheat to map chromosomal locations of genes controlling root hair length. Ann. Bot. 119, 1333-1341

94. Brown, L.K. et al. (2012) What are the implications of variation in root hair length on tolerance to phosphorus deficiency in combination with water stress in barley (Hordeum vulgare). Ann. Bot. 110, 319-328

95. Burridge, J.D. et al. (2019) A case study on the efficacy of root phenotypic selection for edaphic stress tolerance in low-input agriculture: common bean breeding in Mozambique. Field Crops Res. 244, 107612

96. Strock, C.F. et al. (2019) Seedling root architecture and its relationship with seed yield across diverse environments in Phaseolus vulgaris. Field Crop Res. 237, 53-64

97. Thomas, C.L. et al. (2016) High-throughput phenotyping (HTP) identifies seedling root traits linked to variation in seed yield and nutrient capture in field-grown oilseed rape (Brassica napus L.) Ann. Bot. 118, 655-665
98. Xie, Q. et al. (2017) Identifying seedling root architectural traits 611 associated with yield and yield components in wheat. Ann. Bot. 612 119, 1115-1129 Khokhar, J.S. et al. (2019) Juvenile root traits show limited cor- 614 relation with grain yield, yield components and grain mineral 615 composition traits in Indian wheat under hostile soils. Cereal 616 Res. Commun. 47, 362-382

100. Bai, C. et al. (2019) The relationships between seedling root 618 screens, root growth in the field and grain yield for wheat. 619 Plant Soil 440, 311-326

101. Downie, H.F. et al. (2015) Challenges and opportunities for 621 quantifying roots and rhizosphere interactions through imaging 622 and image analysis. Plant Cell Environ. 38, 1213-1232 623

102. Downie, H.F. et al. (2012) Transparent soil for imaging the 624 rhizosphere. PLOS ONE 7, e44276 625

103. Ma, L. et al. (2019) Hydrogel-based transparent soils for root 626 phenotyping in vivo. Proc. Natl. Acad. Sci. U. S. A. 116, 627 11063-11068 628

104. Miguel, M.A. (2015) Phene synergism between root hair length 629 and basal root growth angle for phosphorus acquisition. Plant 630 Physiol. 167, 1430-1439 631

105. White, P.J. (2019) Root traits benefitting crop production in en- 632 vironments with limited water and nutrient availability. Ann. Bot. 633 124, 883-890

106. Clowes, F.A.L. (2000) Pattern in root meristem development in 635 angiosperms. New Phytol. 146, 83-94 636

107. Marzec, M. et al. (2014) The evolutionary context of root epidermis 637 cell patterning in grasses (Poaceae). Plant Signal. Behav. 9, e27972 638

108. Tsai, S.L. etal. (2003) The root epidermis of Echium plantagineum 639 L.: a novel type of pattern based on the distribution of short and 640 long root hairs. Planta 217, 238-244 